\title{
Modified Sleeve Technique in Aortic Valve-Sparing Operation for Marfan Syndrome
}

\author{
Yung-Szu Wu, MD, ${ }^{1}$ Shih-Rong Hsieh, MD, ${ }^{1,2}$ Chung-Chi Wang, MD, ${ }^{1,2}$ \\ and Chung-Lin Tsai, $\mathrm{MD}^{1}$
}

\begin{abstract}
We devised a simple modification of the Florida Sleeve procedure to perform aortic valve-sparing surgery. This technique is simple, quick, effective, and safe. We used this technique in operations performed on two young patients with Marfan syndrome. The initial and short-term results were satisfactory.
\end{abstract}

Keywords: aortic root aneurysm, aortic valve-sparing, Marfan syndrome

\section{Introduction}

Several types of aortic valve-sparing (AVS) operations have been developed and two major types of AVS have been extensively used, including reimplantation of the aortic valve and remodeling of the aortic root. ${ }^{1)}$ In these operations, the wall of the sinus of Valsalva is usually resected and the creation of a neoaortic sinus is necessary. Although the long-term results of these operations are satisfactory, the procedures involved in forming the proper shape of the sinus of Valsalva and precisely tailoring the Dacron graft are complex and not easily reproducible. ${ }^{2,3)}$ A recently introduced technique known as the Florida Sleeve procedure involves wrapping the aortic root aneurysm without resection to preserve the native aortic valve. ${ }^{4,5)}$ We have developed a more simple and reasonable modified version of this method. Moreover, we have performed the new modified technique on

${ }^{1}$ Department of Cardiovascular Surgery, Cardiovascular Center, Taichung Veterans General Hospital, Taichung, Taiwan

${ }^{2}$ National Yang-Ming University School of Medicine, Taipei, Taiwan

Received: November 30, 2017; Accepted: December 15, 2017

Corresponding author: Shih-Rong Hsieh, MD. Department of Cardiovascular Surgery, Cardiovascular Center, Taichung Veterans General Hospital, 1650 Taiwan Boulevard Sect. 4, Taichung 40705, Taiwan, ROC

Email: season@vghtc.gov.tw

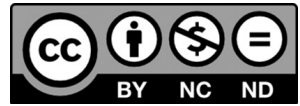

This work is licensed under a Creative Commons Attribution-NonCommercialNoDerivatives International License.

(C2019 The Editorial Committee of Annals of Thoracic and Cardiovascular Surgery two patients with Marfan syndrome. The early and shortterm results are satisfactory.

\section{Case Report}

The first case was a 16-year-old boy with Marfan syndrome. He complained of intermittent palpitation and exercise intolerance for 3 months. His height was $189 \mathrm{~cm}$ and body weight was $47 \mathrm{~kg}$. Echocardiography showed severe mitral regurgitation related to annulus dilatation, cardiomegaly with normal left ventricular function, and aortic root dilatation. Computed tomography angiography (CTA) revealed sinus Valsalva aneurysm with largest diameter of $6.1 \mathrm{~cm}$, sinotubular junction (STJ) was $4.7 \mathrm{~cm}$, diameter of ventriculo-aortic junction (VAJ) was $2.8 \mathrm{~cm}$, and ascending aorta was $3.0 \mathrm{~cm}$ (Fig. 1A). The second case was a 33-year-old woman with Marfan syndrome. She suffered from shortness of breath for 2 months. Echocardiography showed moderate to severe aortic regurgitation, moderate mitral regurgitation, and normal left ventricular function. CTA revealed sinus Valsalva aneurysm with largest diameter of $5.2 \mathrm{~cm}$, STJ was $4.0 \mathrm{~cm}$, diameter of VAJ was $2.5 \mathrm{~cm}$, and ascending aorta was $2.6 \mathrm{~cm}$ (Fig. 1B).

The same procedure was done in both patients. Patients were monitored by transesophageal echocardiography (TEE) during operation. Operation was carried out via a median sternotomy with standard cardiopulmonary bypass through aortic and bicaval cannulations. An intermittent antegrade tepid blood cardioplegia infusion was used for myocardial protection. After aortic 

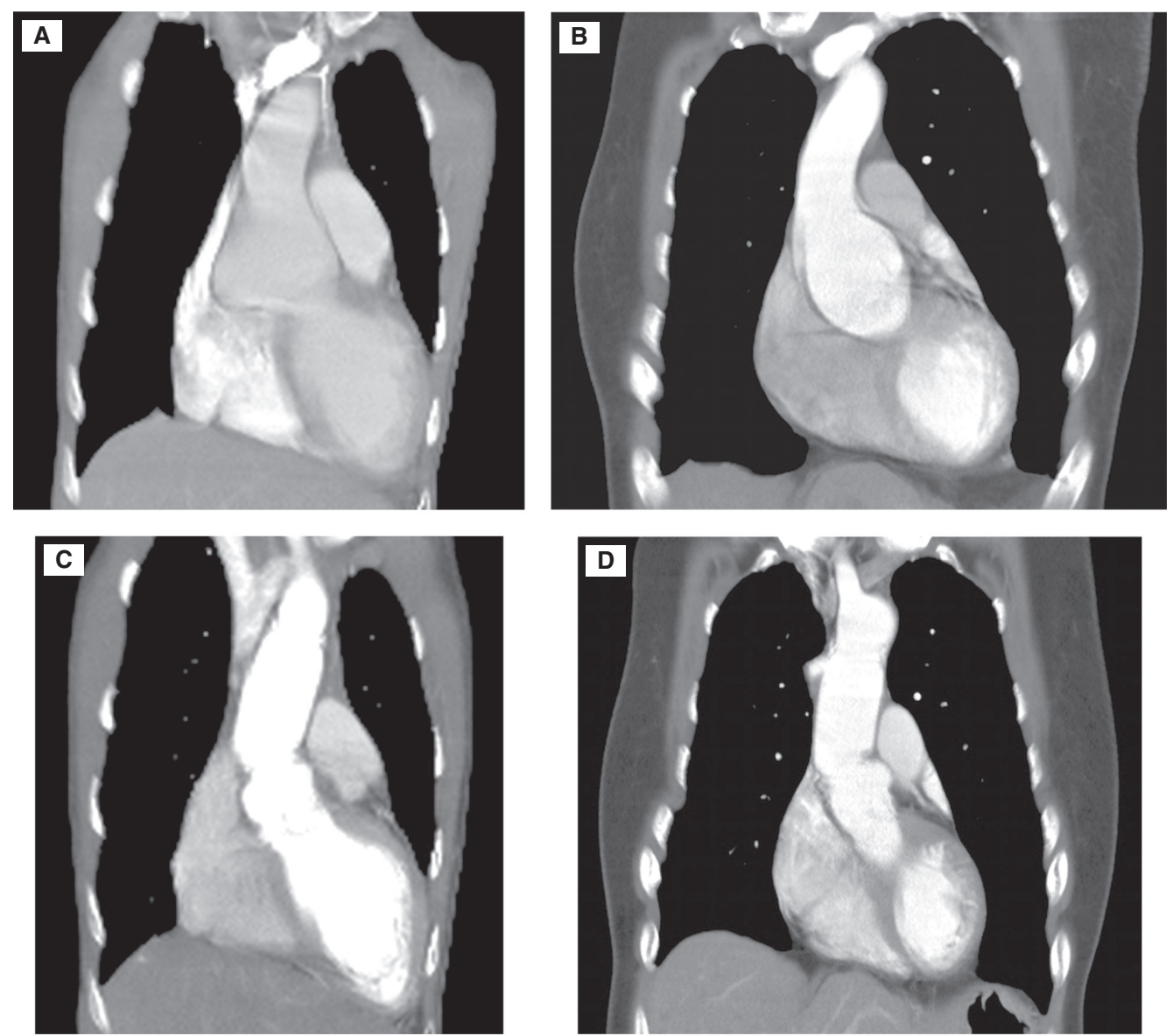

Fig. 1 (A) Preoperative CTA of the first case: sinus Valsalva diameter was $6.1 \mathrm{~cm}$ and ascending aorta was $3.0 \mathrm{~cm}$. (B) Preoperative CTA of the second case: sinus Valsalva diameter was $5.2 \mathrm{~cm}$ and ascending aorta was $2.6 \mathrm{~cm}$. (C) Postoperative CTA of the first case: sinus Valsalva diameter was $3.1 \mathrm{~cm}$, and ascending aorta was $2.9 \mathrm{~cm}$. (D) Postoperative CTA of the second case: sinus Valsavla diameter was $3.2 \mathrm{~cm}$, and ascending aorta was $3.3 \mathrm{~cm}$. CTA: computed tomography angiography

cross-clamping, the ascending aorta was resected and sectioned at the level about $1 \mathrm{~cm}$ above the STJ (Fig. 2D). A left atriotomy along the right interatrial groove was performed. Mitral valve was repaired by posterior annuloplasty with an annuloplasty semi-rigid band (Cosgrove-Edwards, 34 mm, Edwards Lifesciences, LLC). Body temperature was cooled to $20^{\circ} \mathrm{C}$. Distal ascending aorta up to the proximal arch was resected and replaced with a one-branch aortic polyester graft (Gelweave, 26mm, Terumo, Vascutek, UK; Fig. 2E) during deep hypothermic circulatory arrest. Retrograde cerebral perfusion was used for de-airing. After completion of distal aorta anastomosis, the arterial cannulation was shifted to the side branch of the aortic graft. Cardiopulmonary bypass was restarted and the patient was rewarmed.

A Gelweave Valsalva graft (Terumo, Vascutek, UK) measuring approximately $5 \mathrm{~mm}$ larger in size than the
VAJ diameter estimated by preoperative computed tomography (CT) scan was used for reconstruction. The conduit Valsalva graft was marked at three evenly spaced points along the collar line (the position for fixation of the nadirs of the aortic annulus), at three evenly spaced points at the upper margin of the Valsalva portion (for fixation of three commissures), and at two keyhole positions (after confirming the position of bilateral coronary arterial orifices during operation). Two J-shaped lines connected the lateral aspect of the keyholes down to the collar (Fig. 2A). The graft was trimmed leaving only $3-5 \mathrm{~mm}$ of material at the collar and only $10 \mathrm{~mm}$ straight conduit above the sinus ball. These two J-shaped lines were cut using scissors from the collar up to the keyhole marks and the keyholes were created concomitantly (Fig. 2B). External dissection of the aortic root was performed down to the base of the aortic annulus, and the subvalvular plane was externally 
A

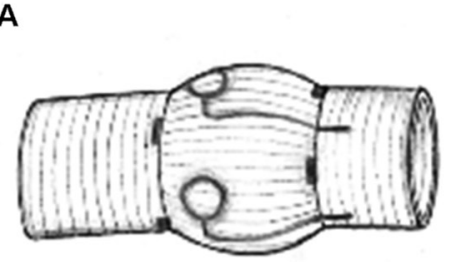

B
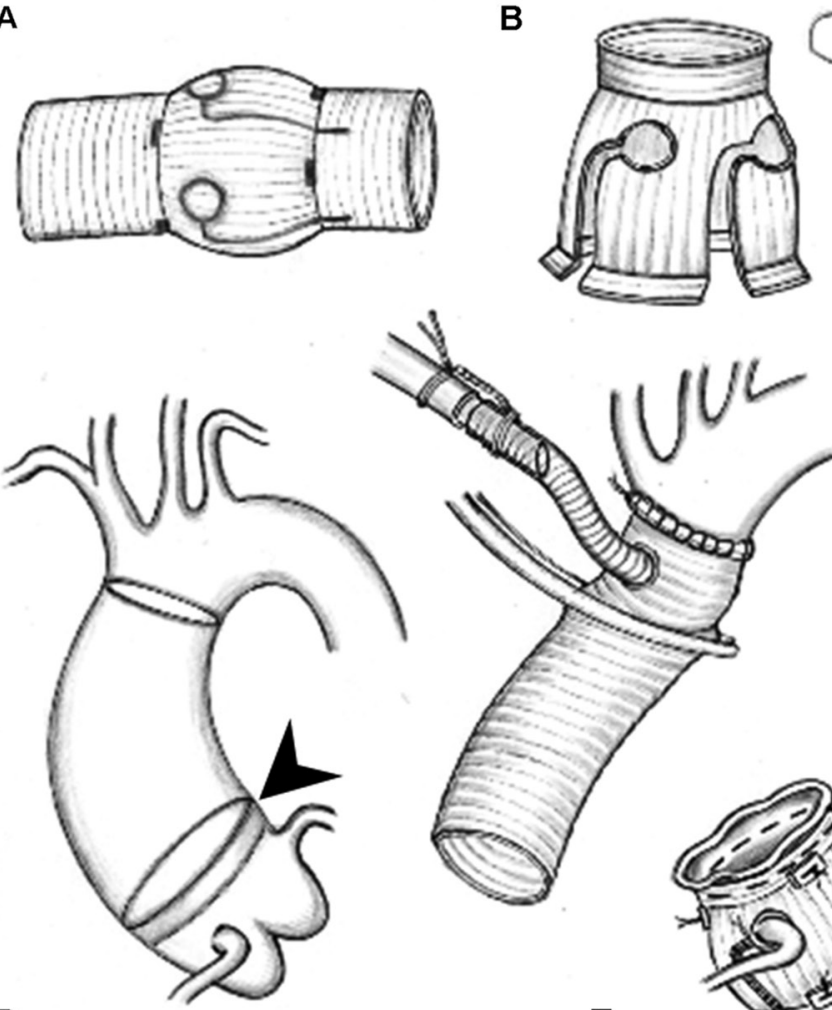

D

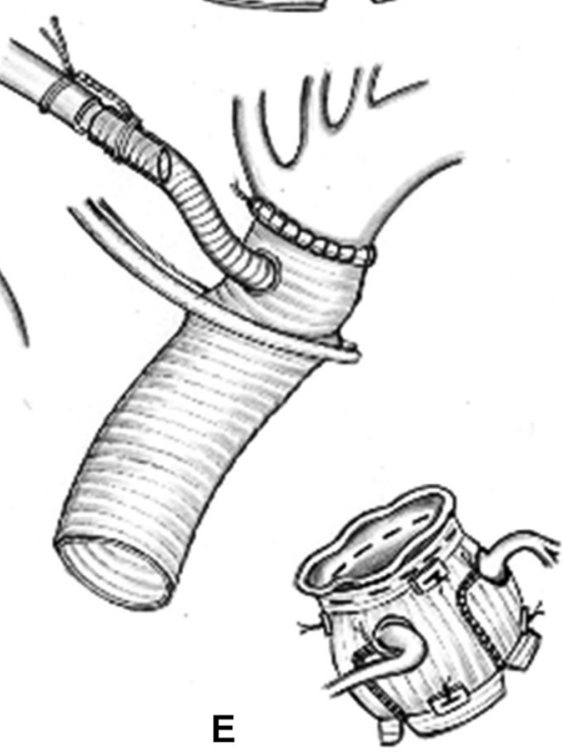

C

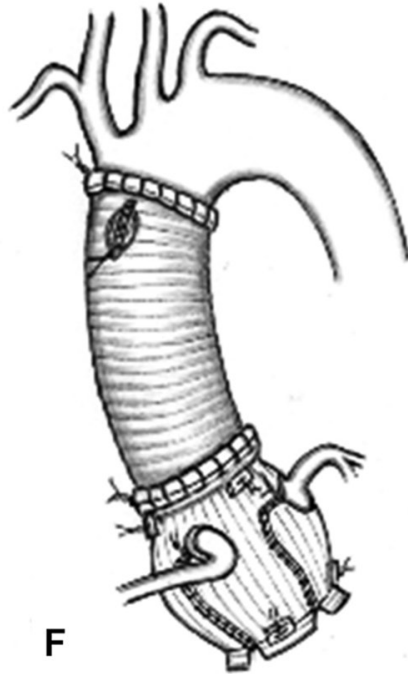

Fig. 2 (A and $\mathbf{B}$ ) showed the J-shape line and keyhole mark for coronary artery on the Valsava graft. (C) showed the position of six anchoring sutures which located at 3 nadirs of the aortic valve annulus and 3 the upper margin of each commissure. (D) showed the resection margin of aortic root. (E) showed the $2 \mathrm{~J}$-shape slit of the Valsava graft were repaired. (F) showed the final shape of ascending aorta graft and the Valsava graft.

freed to the greatest extent that was possible. Six anchoring sutures were used to fix the wrapping graft. Three were located at the VAJ below the three nadirs of the aortic valve annulus, and three at the upper margin of each commissure (Fig. 2C). These sutures were passed through the previously made marks on the graft and knotted. A horizontal mattress of sutures secured the aortic wall to the graft along the level of STJ and for plication of excessive aortic tissue (Figs. 2C and 2E). For the two cases of Marfan syndrome presented herein, we used a narrow piece of Teflon felt to reinforce the inner side of the degenerative aortic wall. Then, the two J-shaped slits of the Valsalva graft were repaired by double rows of 4-O polypropylene running sutures starting from the collar margin and ending at the coronary keyholes (Fig. 2E). The new STJ was anastomosed to the stump of the ascending aorta graft to complete the operation (Fig. 2F). The intraoperative TEE showed minimal aortic regurgitation and mild mitral regurgitation after the procedure.

After operation, the patients recovered without neurologic or cardiac complications. Two months after operation, the follow-up echocardiography showed mild mitral regurgitation and mild aortic regurgitation in both cases (Figs. 3A and 3B). Twelve months later, the CTA of case 1 revealed the sinus Valsalva diameter was $3.3 \mathrm{~cm}$, STJ was $3.3 \mathrm{~cm}$, and the ascending aorta was $2.9 \mathrm{~cm}$ (Fig. 1C). The CTA of case 2 revealed the sinus Valsalva diameter was $3.2 \mathrm{~cm}$, STJ was $2.8 \mathrm{~cm}$, and the ascending aorta was $3.3 \mathrm{~cm}$ (Fig. 1D).

\section{Comment}

In the past two decades, surgical procedures for AVS operation have been repeatedly refined and improved. The techniques developed by David and Yacoub are complex and technically demanding. ${ }^{1)}$ A few years ago, the Florida Sleeve valve-sparing technique was introduced, providing an alternative modality to the David and Yacoub techniques. ${ }^{5)}$ The Sleeve method simplifies the surgical technique and reduces intraoperative complications. Furthermore, it provides more physiologic-like aortic wall stress and might prolong the durability. ${ }^{6)}$ The long-term 

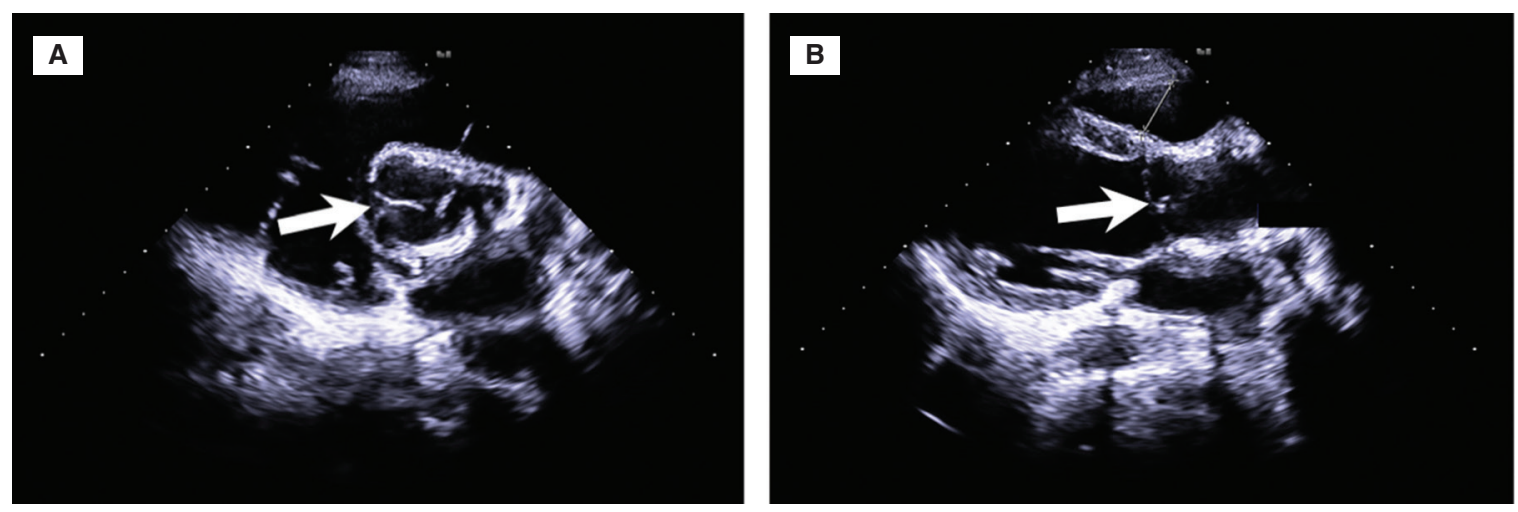

Fig. 3 (A) Postoperative echocardiography of the first case: the aortic valve (white arrow) in parasternal short-axis view. (B) Postoperative echocardiography of the first case: the aortic valve (white arrow) in parasternal long-axis view.

result of Florida Sleeve technique for Marfan patients has been reported that survival rate was $94 \%$ at 1 to 8 year and $100 \%$ free from reoperation in 8 years. ${ }^{7)}$ However, in the Sleeve procedure, a coronary button of inadequate size may compromise blood flow through the coronary ostium. When a root aneurysm is $>6 \mathrm{~cm}$, redundant tissue could create turbulent flow in the remodeled aortic root, which may reduce the durability of the Sleeve technique.

In graft size selection, we supposed that the size of Gelweave graft should be $5 \mathrm{~mm}$ larger than VAJ diameter originally. However, after we had experience of seven cases with modified Sleeve procedure, we prefer to use $32 \mathrm{~mm}$ Gelweave graft in adult patients. If Gelweave graft is smaller than $32 \mathrm{~mm}$, the native aortic wall may create more turbulent flow in new aortic root. Thus, we consider $32 \mathrm{~mm}$ Gelweave graft is suitable for most adult patients.

In our cases, we modified the Florida Sleeve technique to make the surgery simpler. First, only three anchoring sutures were used to provide even fixation of the Valsalva graft collar line to the VAJ. Three additional commissure anchoring sutures were placed to fix the commissures to the Valsalva graft evenly. The position of each suture was easy to define and the graft was fixed into proper position immediately. Thus, the operation was simple, and avoided injury to the conduction system. Second, we use a J-shaped incision to create the coronary keyhole. The slits of the prosthesis can be repaired with ease and without placing sutures directly below the coronary artery. Moreover, it is easy to adjust the size and position of the keyholes. If the coronary artery is compressed after the heartbeat is restored, the keyhole can be enlarged in any direction by making a few small cuts at the margin of the keyholes. The modified Sleeve technique preserves the native aortic root wall and indeed avoids bleeding from aortic root sutures. In conclusion, the initial results of our technique were satisfactory. A larger number of cases and long-term follow-up are necessary to prove its durability.

\section{Disclosure Statement}

No financial interests or potential conflicts of interest.

\section{References}

1) David TE. Aortic valve sparing operations: a review. Korean J Thorac Cardiovasc Surg 2012; 45: 205-12.

2) David TE. Aortic valve sparing in different aortic valve and aortic root conditions. J Am Coll Cardiol 2016; 68: 654-64.

3) Arabkhani B, Mookhoek A, Di Centa I, et al. Reported outcome after valve-sparing aortic root replacement for aortic root aneurysm: a systematic review and meta-analysis. Ann Thorac Surg 2015; 100: 1126-31.

4) Hetzer R, Komoda T, Komoda S, et al. New aortic root remodeling surgery in aortic root aneurysm. Ann Thorac Surg 2010; 89: 1260-4.

5) Komoda T, Komoda S, Gehle P, et al. Reverse graft placement in the Florida sleeve procedure for aortic root aneurysm. Ann Thorac Surg 2013; 95: 723-5.

6) Tasca G, Selmi M, Votta E, et al. Aortic root biomechanics after Sleeve and David sparing techniques: a finite element analysis. Ann Thorac Surg 2017; 103: 1451-9.

7) Aalaei-Andabili SH, Martin T, Hess P, et al. Florida Sleeve procedure is durable and improves aortic valve function in marfan syndrome patients. Ann Thorac Surg 2017; 104: 834-9. 